\title{
Invariance Property of the $\mu$ Stability Test with respect to the Characteristic Impedance
}

\author{
Amr A. Ibrahim, ${ }^{1}$ Ali M. Darwish, ${ }^{1}$ and H. Alfred Hung ${ }^{2}$ \\ ${ }^{1}$ Electronics Engineering Department, American University in Cairo, Cairo, Egypt \\ ${ }^{2}$ Army Research Laboratory, Adelphi, MD 20783, USA \\ Correspondence should be addressed to Amr A. Ibrahim, amralaa87@gmail.com
}

Received 21 December 2011; Revised 18 February 2012; Accepted 20 February 2012

Academic Editor: Chien-Jen Wang

Copyright ( $) 2012$ Amr A. Ibrahim et al. This is an open access article distributed under the Creative Commons Attribution License, which permits unrestricted use, distribution, and reproduction in any medium, provided the original work is properly cited.

\begin{abstract}
Stability of electrical amplifiers is of crucial importance. Among the popular stability tests is the $\mu$-test which has many advantages over other tests like the $K-\Delta$ test. However, the value of $\mu$ parameter is dependent on the input/output terminal characteristic impedance $Z_{o}$ used and this raises the concern that the predictions of the test are dependent on the choice of $Z_{o}$. This paper proves that the conclusions of the $\mu$-test regarding stability/instability remain invariant with $Z_{o}$. This proof is necessary for gaining confidence in the results of the $\mu$-test and should benefit circuit designers. Similar proofs should be extended to all other stability tests for additional insights into their validity under different circuit termination.
\end{abstract}

\section{Introduction}

The stability of a microwave amplifier, or equivalently its resistance to oscillations, is one of the main concerns when designing active microwave circuits. This classical problem of stability of linear two-port network has been intensively investigated in the technical literature over the last fifty years [1-7]. Several sets of conditions were proposed to check stability. All these sets of conditions, which were derived by steady state analysis, have been shown to be equivalent [16]. These tests determine whether it is possible to find a set of passive terminations that will cause the terminated two-port network to have unstable poles given that the network parameters do not have right-hand poles (Rollett's proviso) [3]. If there are no such terminations, the twoport network is unconditionally stable. Among these tests, the most popular are the $K-\Delta$ test [3] and the $\mu$-test [5]. These two tests are widely used in many CAD programs and textbooks discussing the design of both amplifiers as well as oscillators $[8,9]$. The $K-\Delta$ relies on two conditions to assess the stability of microwave amplifiers. On the other hand, the $\mu$-test relies only on one parameter to assess the stability of the amplifier circuit. Usually, the $\mu$-test is calculated from the $S$-parameters of the circuits under test. However, in circuits composed of lumped components, the choice of $Z_{o}$ is ambiguous and hence the $\mu$-test value may change based on the value of $Z_{o}$. In this paper, it is proven that the $\mu$ test result does not depend on the choice $Z_{o}$, as long as $Z_{o}$ takes real positive values. Meaning that if the circuit is stable for a certain $Z_{o}$ then it will be also stable for any other $Z_{o}$ (i.e., $\mu$ value will always be greater than one), and vice versa. Moreover, it is shown that the worst case condition ( $\mu$ value $\sim 1$ ) will occur, when $Z_{o}$ tends to zero or infinity. One of the benefits of this analysis is that the $\mu$-test can also be applied to circuits composed of lumped components with arbitrary $Z_{0}$.

Some stability tests that vary with $Z_{o}$ can yield misleading results. For example, a common oscillation test is to evaluate the round trip gain $\left|\Gamma_{\text {in }} \Gamma_{S}\right|$. The test requires that $\left|\Gamma_{\text {in }} \Gamma_{S}\right|>$ 1 for oscillations [9], where $\Gamma_{\text {in }}$ is the input reflection coefficient of the oscillator circuit and $\Gamma_{S}$ is the source reflection coefficient as shown in Figure 1. Suppose we have a negative resistance oscillator with input impedance of -25 $+\mathrm{j} 10 \mathrm{Ohm}$ and source impedance of $50-\mathrm{j} 10 \mathrm{Ohm}$. Then $\left|\Gamma_{\text {in }} \Gamma_{S}\right|$ may be plotted, as a function of $Z_{o}$ as in Figure 2. It is clear that $\left|\Gamma_{\text {in }} \Gamma_{S}\right|>1$ for small values of $Z_{o}$, and $\left|\Gamma_{\text {in }} \Gamma_{S}\right|<$ 1 otherwise. The variance of $\left|\Gamma_{\text {in }} \Gamma_{S}\right|$ with $Z_{o}$ makes the test's conclusions questionable. Thus, the invariance of a stability 


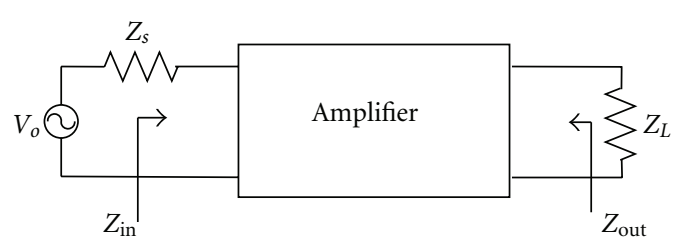

FIgURE 1: The amplifier circuit.

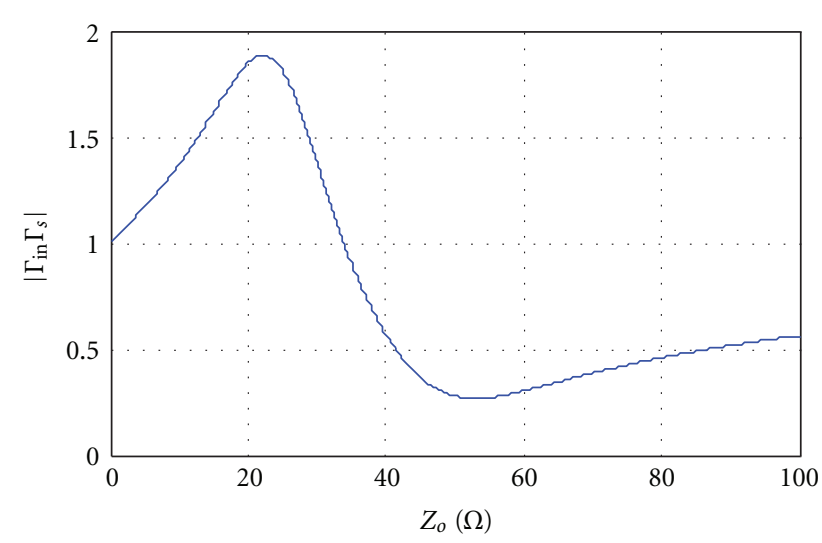

Figure 2: The round trip gain $\left|\Gamma_{\text {in }} \Gamma_{S}\right|$ as a function of $Z_{o}$ used in the evaluation of $\Gamma_{\text {in }}$ and $\Gamma_{S}$.

test with $Z_{o}$ is crucial and cannot be assumed without a proof. This paper addresses this concern for the popular $\mu$ test.

\section{Mathematical Treatment}

In this section, it is proven that the $\mu$-test does not depend on the choice of $Z_{o}$ of terminal ports. It is convenient to start the proof from the impedance domain $(Z)$ since it does not depend on the choice of $Z_{0}$. For an amplifier circuit to be stable (according to the $\mu$-test), the condition

$$
\left|\Gamma_{\text {in }}\right|<1
$$

must be satisfied for any passive load termination $\left(Z_{L}\right)$ [5]. The $\mu$-test defines a parameter, $\mu$, such that

$$
\mu=\frac{1}{\left|\Gamma_{\max }\right|},
$$

where $\left|\Gamma_{\max }\right|$ is the maximum reflection coefficient (for any possible load). If $\left|\Gamma_{\max }\right|<1$, then the network is stable, $\mu$ is greater than 1, and no negative impedances can be produced, and vice versa.

The input impedance of the amplifier circuit is given by

$$
Z_{\text {in }}=Z_{11}-\frac{Z_{12} Z_{21}}{Z_{L}+Z_{22}}=\frac{Z_{11} Z_{L}+\Delta_{Z}}{Z_{L}+Z_{22}}
$$

where $\Delta_{Z}$ is the determinant of the impedance matrix.

Equation (3) has the form of a bilinear transformation. The bilinear transformation is a complex mapping technique and has the property of mapping circles in one domain into circles in another domain with lines as the limiting case. Under this transformation, the imaginary axis in the $Z_{L}$ domain $\left(\operatorname{Re}\left(Z_{L}\right)=0\right)$ is mapped into a circle in the $Z_{\text {in }}$ domain as shown in Figure 3 with centre $C_{\text {in }}$ and radius $R_{\text {in }}$ given by

$$
\begin{gathered}
C_{\text {in }}=\frac{2 Z_{11} \operatorname{Re}\left(Z_{22}\right)-Z_{12} Z_{21}}{2 \operatorname{Re}\left(Z_{22}\right)}, \\
R_{\text {in }}=\left|\frac{Z_{12} Z_{21}}{2 \operatorname{Re}\left(Z_{22}\right)}\right| .
\end{gathered}
$$

For the amplifier to be absolutely stable, the mapped circle in the $Z_{\text {in }}$ domain must lie completely in the right half plane or

$$
\operatorname{Re}\left(C_{\text {in }}\right)>R_{\text {in }} \text {. }
$$

Furthermore, the greater the inequality $\operatorname{Re}\left(C_{\text {in }}\right)>R_{\text {in }}$, the higher the stability. In the previous equation, it was assumed that the region $\left(\operatorname{Re}\left(Z_{L}\right)>0\right)$ is mapped to the interior of the circle in the $Z_{\text {in }}$ domain which will happen given that the condition $\left(\operatorname{Re}\left(Z_{22}\right)>0\right)$ is satisfied.

Now assuming that (5) is satisfied and the amplifier is absolutely stable in the $Z_{\text {in }}$ domain, one can map this circle to the $\Gamma_{\text {in }}$ domain using the bilinear transformation

$$
Z_{\text {in }}=\frac{Z_{o} \Gamma_{\text {in }}+Z_{o}}{-\Gamma_{\text {in }}+1} .
$$

For a circle having a centre $\left(C_{\text {in }}\right)$ and radius $\left(R_{\text {in }}\right)$ in the $Z_{\text {in }}$ domain, the mapped circle in the $\Gamma_{\text {in }}$ domain will have a center $\left(C_{\Gamma}\right)$ and radius $\left(R_{\Gamma}\right)$ given by

$$
\begin{gathered}
C_{\Gamma}=\frac{\left|C_{\text {in }}\right|^{2}-R_{\text {in }}^{2}-Z_{o}^{2}+j 2 Z_{o} \operatorname{Im}\left(C_{\text {in }}\right)}{\left|C_{\text {in }}\right|^{2}-R_{\text {in }}^{2}+Z_{o}^{2}+2 Z_{o} \operatorname{Re}\left(C_{\text {in }}\right)}, \\
R_{\Gamma}=\frac{2 R_{\text {in }} Z_{o}}{\left|C_{\text {in }}\right|^{2}-R_{\text {in }}^{2}+Z_{o}^{2}+2 Z_{o} \operatorname{Re}\left(C_{\text {in }}\right)} .
\end{gathered}
$$

Since the interior of the circle in the $Z_{\text {in }}$ domain is mapped to the interior of the circle in the $\Gamma_{\text {in }}$ domain, (1) can be rewritten as

$$
\left|C_{\Gamma}\right|+R_{\Gamma}<1 .
$$

To check this condition, define

$$
C_{\text {in }}=a+j b .
$$

From (5), one can write

$$
a^{2}=R_{\text {in }}^{2}+\delta^{2},
$$

where $\delta^{2}>0$ is a parameter that can indicate higher stability. Substituting with (10) in (7), one can write

$$
\left|C_{\Gamma}\right|+R_{\Gamma}=\frac{\sqrt{\left(b^{2}+\delta^{2}+Z_{o}^{2}\right)^{2}-\left(2 \delta Z_{o}\right)^{2}}+2 R_{\text {in }} Z_{o}}{\left(b^{2}+\delta^{2}+Z_{o}^{2}\right)+2 a Z_{o}} .
$$

In this case, the $\mu$-test is simply the inverse of the previous expression. Thus, based on (2),

$$
\mu=\frac{1}{\left|C_{\Gamma}\right|+R_{\Gamma}}=\frac{\left(b^{2}+\delta^{2}+Z_{o}^{2}\right)+2 a Z_{o}}{\sqrt{\left(b^{2}+\delta^{2}+Z_{o}^{2}\right)^{2}-\left(2 \delta Z_{o}\right)^{2}}+2 R_{\text {in }} Z_{o}} .
$$




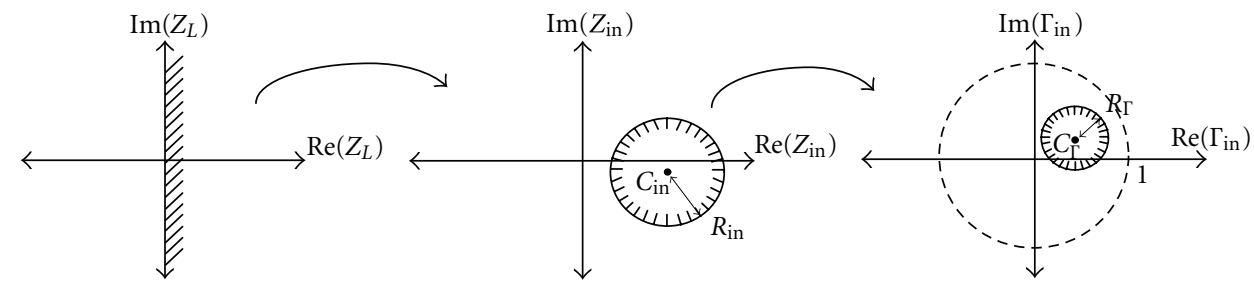

Figure 3: Mapping from the $\left(Z_{L}\right)$ impedance plane into $Z_{\text {in }}$ plane followed by mapping into $\Gamma_{\text {in }}$ plane.

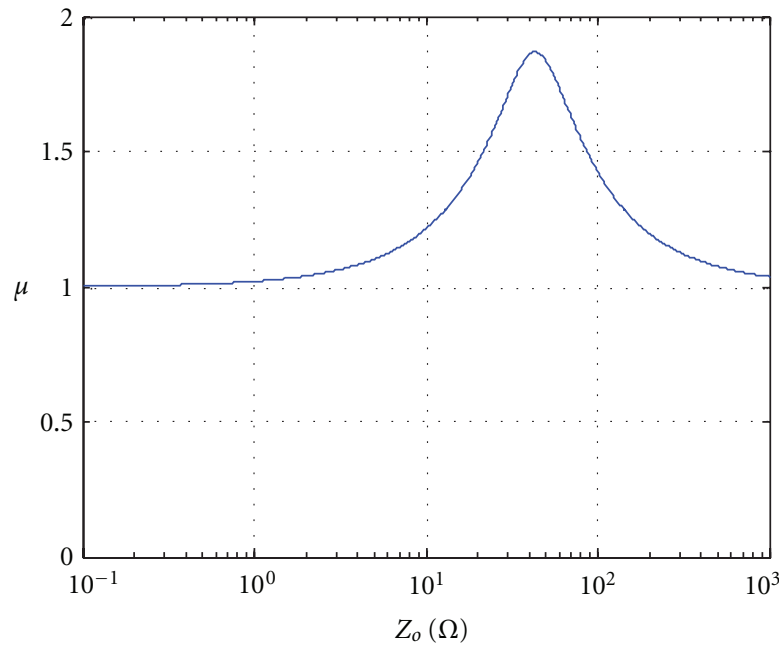

(a)

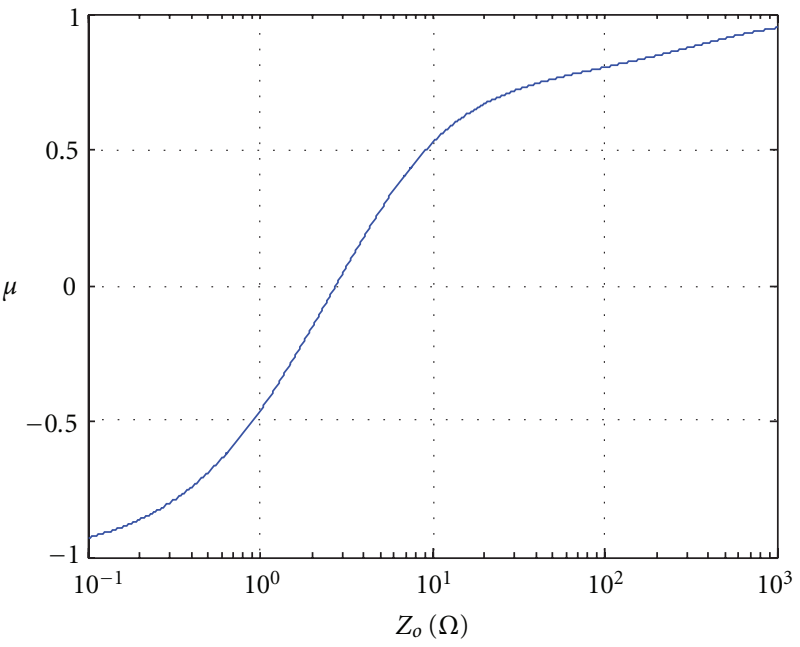

(b)

FIgURE 4: $\mu$-test as a function of $Z_{o}$ for (a) unconditionally stable amplifier and (b) conditionally stable amplifier.

The value of the previous expression is always greater than 1 given that (5) is satisfied irrespective of the value of $Z_{o}$. That is because the first term in the numerator is greater than the first term in the denominator, and the same is true for the second term in the numerator and denominator. Also, at the stability edge in the $Z_{\text {in }}$ domain where $\delta \rightarrow 0$, the value of $\mu$ will approach 1 . It should be noted that the value of $\mu$ will also approach 1 in the limiting case as $Z_{o}$ approaches zero or infinity. The previous steps of the proof are summarized in Figure 3.

\section{Verification of Theory Using Two Examples}

Two examples are given to validate the previous results. The first example is for an unconditionally stable amplifier and the second one is for a conditionally stable amplifier. First, consider the parameters of an amplifier circuit in the $Z$ domain at certain frequency $\left(f_{o}\right)$ as

$$
Z_{\text {amp }}=\left[\begin{array}{cc}
36-j 24 & 1.5-j 4 \\
-15-j 585 & 20-j 28
\end{array}\right],
$$

which corresponds to the following $S$-parameters (in a 50 Ohms system):

$$
S_{\text {amp }}=\left[\begin{array}{cc}
0.1 \angle-10.5^{\circ} & 0.05 \angle-46.5^{\circ} \\
7 \angle-68.5^{\circ} & 0.15 \angle-112.7^{\circ}
\end{array}\right] .
$$

For this amplifier, the $\mu$-test, given by (15), is calculated for different values of $Z_{o}$ and the result is shown in Figure 4(a),

$$
\mu=\frac{1-\left|S_{11}\right|^{2}}{\left|S_{22}-\Delta_{S} \overline{S_{11}}\right|+\left|S_{12} S_{21}\right|},
$$

where $\Delta_{S}$ is determinant of the $S$-parameters matrix, and the upper bar indicates conjugation. It is clear from Figure 4(a) that the value of $\mu$ is always greater than 1 and it approaches 1 as $Z_{o}$ tends to zero or infinity. The same conclusion can be obtained by observing the mapped circles of the region $\left(\operatorname{Re}\left(Z_{L}\right)>0\right)$ in the $\Gamma_{\text {in }}$ domain as a function of $Z_{o}$ (Figure 5(a)), where all the mapped circles are located inside 


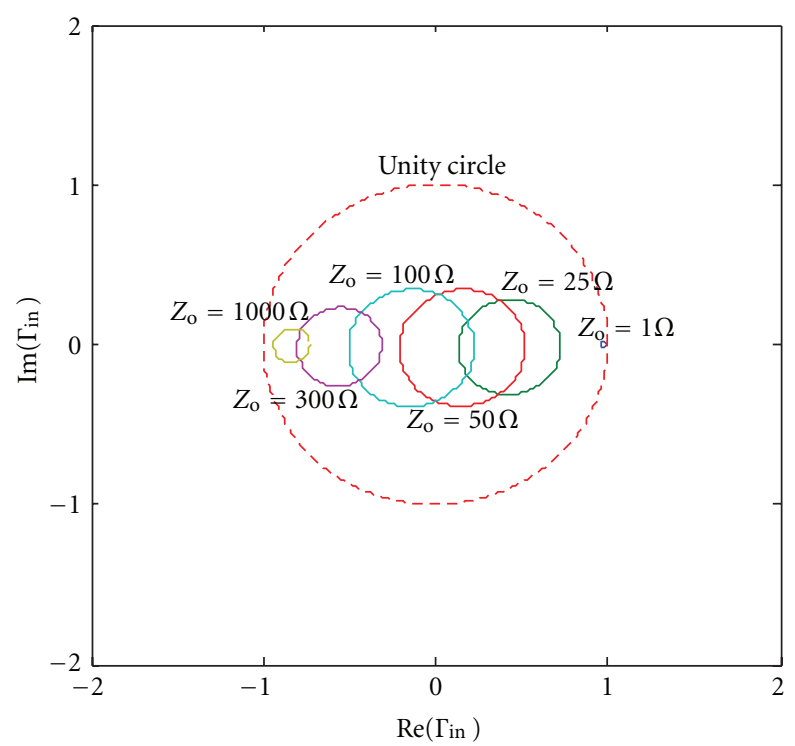

(a)

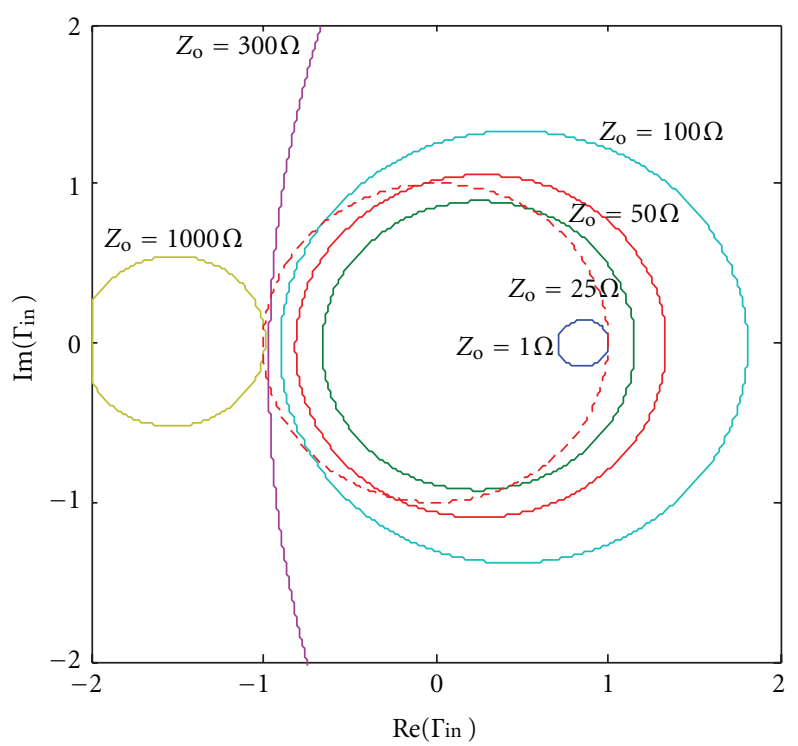

(b)

Figure 5: Mapped circles in the $\Gamma_{\text {in }}$ domain for (a) unconditionally stable amplifier and (b) conditionally stable amplifier.

the unity circle $\left(\left|\Gamma_{\text {in }}\right|=1\right)$. Now consider a second case where the amplifier $Z$-parameters are changed to

$$
Z_{\text {amp }}=\left[\begin{array}{cc}
1.8-j 31 & 0.87-j 8.4 \\
-115.6-j 373.3 & -9.4-j 30.6
\end{array}\right]
$$

which corresponds to the following $S$-parameters (in a 50Ohms system):

$$
S_{\text {amp }}=\left[\begin{array}{cc}
0.1 \angle-11.3^{\circ} & 0.15 \angle-45.9^{\circ} \\
7 \angle-68.8^{\circ} & 0.15 \angle-114.7^{\circ}
\end{array}\right] .
$$

The $\mu$-test as a function of $Z_{o}$ is shown in Figure 4(b). As the figure indicates, the value of $\mu$ is less than 1 , irrespective of $Z_{o}$. Also, Figure 5(b) shows that the mapped circles of the region $\left(\operatorname{Re}\left(Z_{L}\right)>0\right)$ in the $\Gamma_{\text {in }}$ domain intersect with the unity circle at all values of $Z_{o}$. The previous two examples illustrate the application of the theory.

\section{Conclusion}

One of the most popular tests for stability is the $\mu$-test due to its simplicity (one parameter, instead of two) and its ability to indicate relative stability. This paper proves that the conclusions reached through the $\mu$-test are invariant with characteristic impedance $Z_{o}$ choices. This proof is necessary for using the $\mu$-test with confidence. A stability test which does not meet this condition may lead to inaccurate conclusions. Two examples illustrating the application of the theory were provided.

\section{References}

[1] D. Youla, "A Note on the stability of linear nonreciprocal Nports," Proceedings of the IRE, vol. 48, pp. 121-122, 1960.
[2] D. Woods, "Reappraisal of the unconditional stability criteria for active 2-port networks in terms of S parameters," IEEE Trans Circuits Syst, vol. 23, no. 2, pp. 73-81, 1976.

[3] J. Rollett, "Stability and power-gain invariants of linear twoports," IRE Transactions on Circuit Theory, vol. 9, no. 1, pp. 29-32, 1962.

[4] F. Llewellyn, "Some fundamental properties of transmission systems," Proceedings of the IRE, vol. 40, no. 3, pp. 271-283, 1952.

[5] M. L. Edwards and J. H. Sinsky, "A new criterion for linear 2port stability using a single geometrically derived parameter," IEEE Transactions on Microwave Theory and Techniques, vol. 40, no. 12, pp. 2303-2311, 1992.

[6] R. W. Jackson, "Rollett proviso in the stability of linear microwave circuits-a tutorial," IEEE Transactions on Microwave Theory and Techniques, vol. 54, no. 3, pp. 993-1000, 2006.

[7] C. C. Meng, H. J. Wei, and P. H. Sun, "Criteria for the evaluation of linear two-port stability using two geometrically derived parameters," International Journal of Microwave and Wireless Technologies, vol. 1, no. 1, pp. 65-72, 2009.

[8] A. Suarez, Analysis and Design of Autonomous Microwave Circuits, Wiley-IEEE Press, Hoboken, NJ, USA, 2009.

[9] P. L. D. Abrie, Design of RF and Microwave Amplifiers and Oscillators, Artech House, Boston, Mass, USA, 1999. 

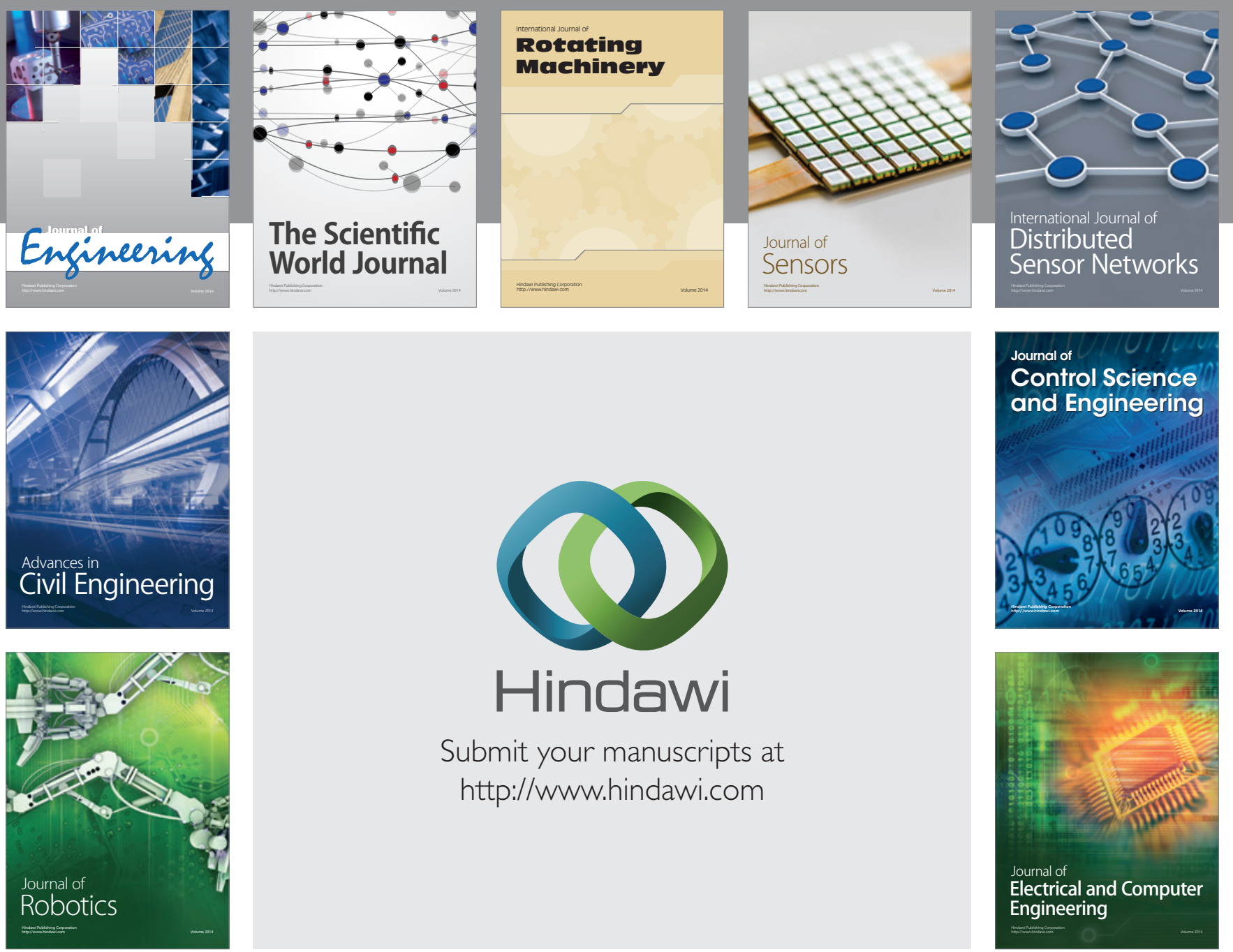

Submit your manuscripts at

http://www.hindawi.com
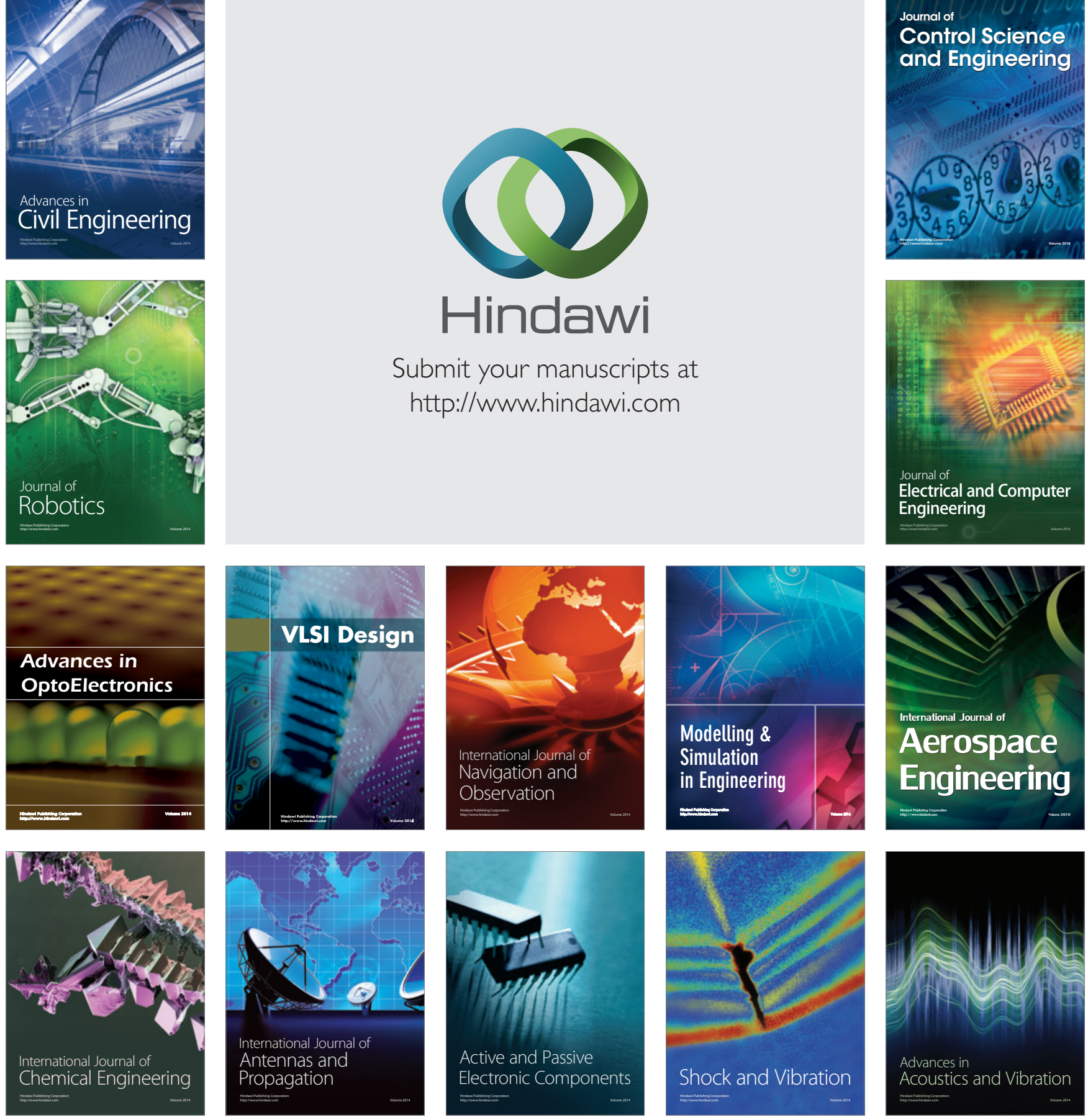